\title{
ESI: Easy software interfacing tools for Turbo Pascal programming of digital input-output devices
}

\author{
JONATHAN SCHULL and EVAN M. KAMINER \\ Haverford College, Haverford, Pennsylvania
}

\begin{abstract}
We describe some programming tools for real-time monitoring and control of digital laboratory devices. These create several interrupt-driven processes that operate in the "background" of the user's program. An event buffer logs switch closures, thus helping the user's program keep up with events. Other simple commands (e.g., TurnOn) take a line number and a time argument as parameters; they are automatically executed at the time specified. Applications to operant conditioning are discussed.
\end{abstract}

This paper describes a set of programming tools that are intended to allow novice Pascal programmers to write laboratory control programs using IBM compatible computers. The Easy Software Interface (ESI, pronounced "easy") was originally developed for use in operant conditioning experiments and is described here in the context of that application. However, ESI should be useful in any applications involving real-time monitoring and control of digital input events (e.g., switch closures) and digital output events (e.g., relay operations).

The programmer of any real-time experiment must ensure that short-lived input events do not go undetected (input monitoring) and that output events are executed at appropriate times (output scheduling). However, in order to react rapidly to an input event (or to avoid missing it entirely), one must frequently check input lines for changes of status (polling), and this limits the amount of time that can be spent doing other tasks. Similarly, while a program is counting down the time until a relay needs to be operated, it cannot also check input lines.

The technical solution to this dilemma is a program that jumps back and forth between input monitoring, output scheduling, and other necessary tasks (e.g., computation of time, decision making, and data logging) frequently enough that no process lags too far behind. Mediating and coordinating these competing activities is a complex problem. On mainframe computers with time-sharing operating systems, users can write quasi-independent programs that handle each task separately, sharing data as needed. On personal computers running under non-timesharing operating systems (e.g., IBM PC compatibles nunning under Microsoft's disk-operating system, MS-DOS), responsibility for mediating and coordinating multiple

\footnotetext{
We wish to thank Tom Lacki, who assisted in the development of a precursor to the present system; Jon Bondy for consultation and criticism; and Lamont Jamison, Ed Myers, David Pine, Beatrice Wood, Richard Bellon, and Julin Maloof. We also thank Haverford College for supporting the research that necessitated development of this software. Address reprint requests to Jonathan Schull, Department of Psychology, Haverford College, Haverford, PA 19041
}

processes of this sort falls to the applications program. Such a program is difficult to write, read, and/or modify. ESI simplifies the programmer's task by creating timesharing-like processes that take care of input monitoring, output scheduling, and timing. The individual programmer (or user) is thus freed to concentrate on routines for decision making and data storage. The user's program is typically much simpler and shorter than it would otherwise be, and is more like a description of an experiment than like a description of what the computer must do to implement the experiment. In addition, the ESI system is based upon Borland International's Turbo Pascal, which is generally considered one of the friendliest and most efficient programming environments available on personal computers (Kaplan, 1985). Borland also offers a variety of additional products, called software "toolboxes," for graphics and other applications, which can easily be combined with the ESI routines.

\section{OVERVIEW}

As illustrated in Figure 1, an application program using ESI can be broken down into five logical modules.

1. The User Program contains the experiment-specific routines and, via a single command, specifies the operating parameters of the other modules. The most important of these parameters is the period, the interval between successive checks of the interface board for changes in line status (events). Shorter periods ensure detection of shorter duration events; however, because polling of the interface board briefly interrupts the user program, shorter periods can slow the user program slightly. The optimal period depends upon the user's application.

2. The Timer maintains ESI's software clock and triggers two interrupt-driven routines that operate in the background of the user program: polling of the interface and execution of previously scheduled tasks.

3. The Event Buffer is analogous to a keyboard buffer that allows one to "type ahead" of a word processor that would otherwise be too slow to keep up; ESI's event 


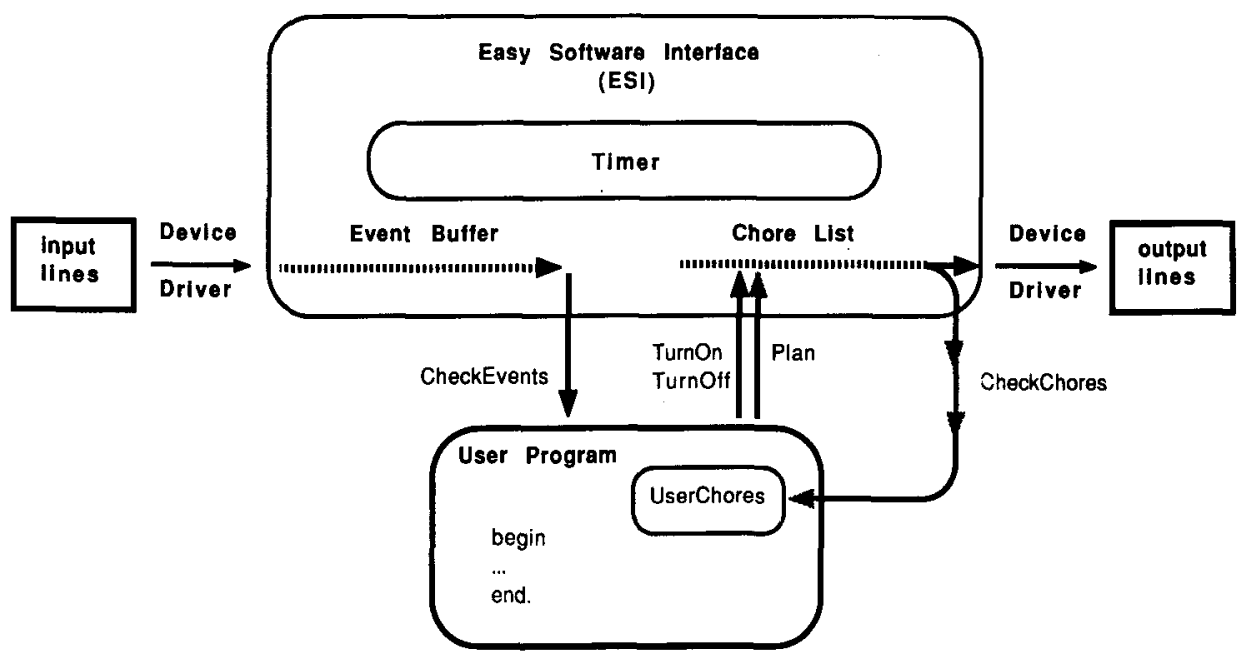

Figure 1. The ESI system. Square-edged boxes denote interfacing hardware. The ESI routines create the data structures called the Event Buffer, the Timer, and the Chore List. The User Program interacts with these data structures through a few simple commands, such as CheckEvents, TurnOn, and TurnOff.

buffer similarly maintains a sequential list of events, as well as the times at which they occurred. This prevents data loss when the user program cannot process events as rapidly as they occur. The polling process puts event data in the buffer at the rate of detection, which allows the user program to take the data out at its own pace, using the procedure CheckEvents. CheckEvents retrieves information about the earliest as-yet-unattended event.

4. The Chore List simplifies the scheduling and execution of delayed tasks, or chores. When writing realtime programs, it is often necessary to perform a task some time in the future. For example, if a feeder is turned on, it will have to be turned off again in about $55 \mathrm{msec}$. In a conventional procedural language, a program would ordinarily need to suspend other activities, count down $55 \mathrm{msec}$, turn off the feeder, and then resume other activities. With ESI, the user program can merely specify the time at which a task should occur in the future; for example,

\section{TurnOff( Feeder, FromNow( 55 ))}

Fifty milliseconds later, ESI does it.

TurnOn and TurnOff are intrinsic chores that are automatically executed at the appropriate time; the Chore List can also be used for delayed execution of user-written chores (UserChores) via the commands Plan and CheckChores, discussed later.

5. The device driver comprises routines specific to a particular interface board. This is the only module that needs to be modified in order to adapt ESI to a new interface card. Our lab uses the DAISI card, manufactured by the Interactive Structures Corporation (Malvern, Pennsylvania), for which we have written a device driver (contained in the file called DAISI.inc, in the following example).

\section{AN EXAMPLE PROGRAM}

The program listed in Figure 2 illustrates a simple User Program with three basic parts. The first part uses com- piler directives to direct compilation of the user program plus the ESI routines into a machine-executable application program. The second part defines useful constants and procedures for the user. The third part is the main program block, which is made up of commands to initialize the ESI system and the basic operation loop of the user program. We discuss some basic features of the Pascal programming language, describe the workings of the main part of the program, and then describe the syntax of the ESI commands.

Turbo Pascal allows the insertion of "(*\$I...*)" directives into the user program to instruct the compiler to include other disk files as part of the user program. By including several ESI files, the user gains access to a few new commands that provide what we think are the essentials for real-time experimentation. These commands can then be combined with the standard Turbo Pascal commands and environment to build powerful modular programs for a variety of purposes. Such programs are also relatively readable, since Pascal encourages the use of English-like names for constants, variables, and procedures. Thus in the CONST statement at the beginning of the program, device names are assigned to numbers corresponding to the lines on the interface card connected to those devices; henceforth, the devices can be referred to by name. ESI similarly defines constants with names, such as "msec," "sec," and "min," which enable users to specify time parameters in the form " $2 *$ min" rather than " 1800 ." Meaningful procedure names are also standard in Pascal, as in the five lines beginning "procedure Reward," which establish the word "Reward" as a command to briefly operate a food dispenser and write a report to the screen.

These constant and procedure names are then used in the main part of the program to specify the contingencies of an experiment on delay of reinforcement in two twobar Skinner boxes. In each box, presses to the left bar are rewarded immediately, and presses to the right bar are rewarded after a 3-sec delay. These contingencies are 
Program DelayedRewards;

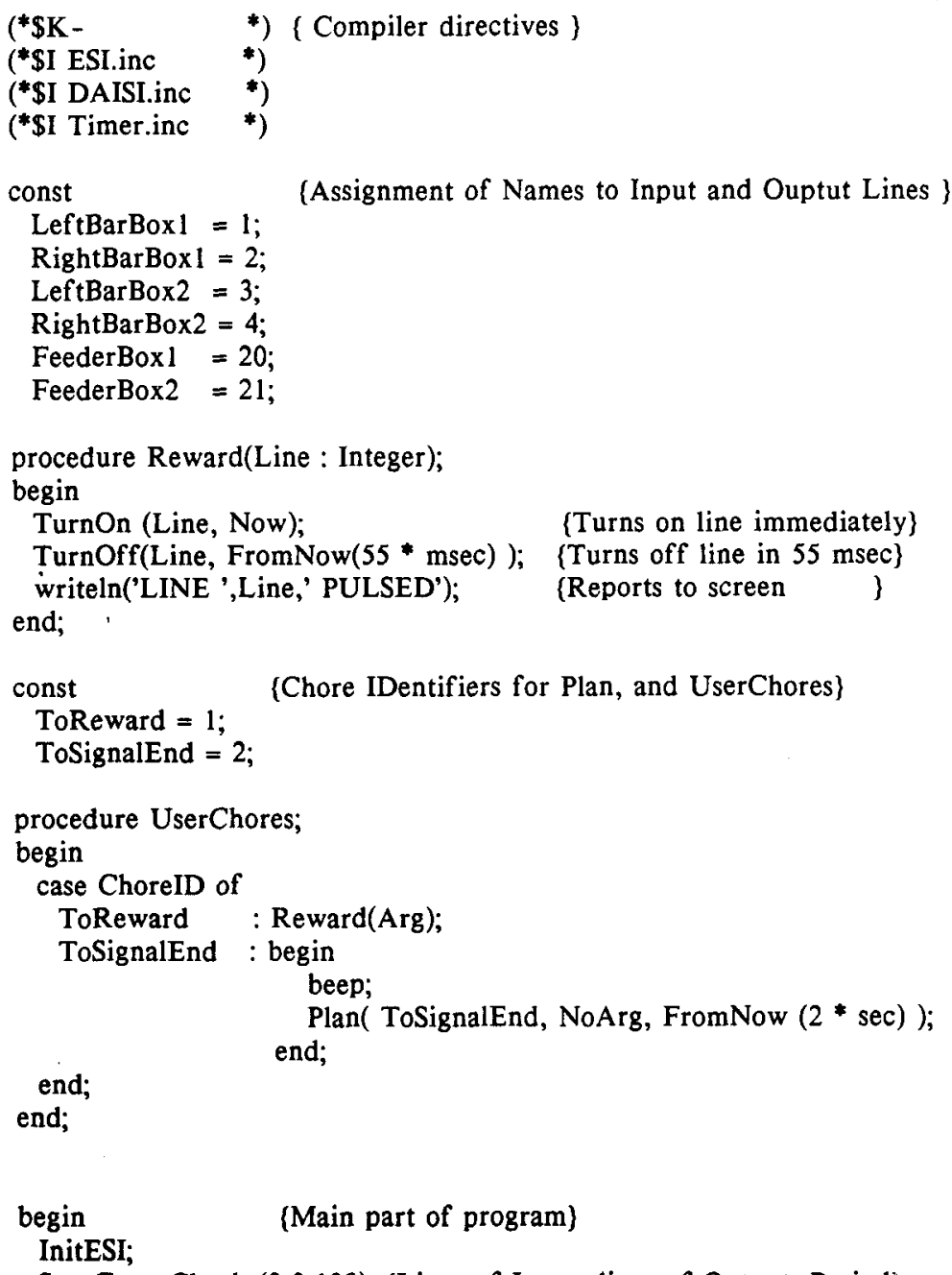

StartEventChecks $(8,8,100)$; (Lines of Input, lines of Output, Period)

Plan( ToSignalEnd, FromNow( $29 * \mathrm{~min}$ ), NoArg );

EndSession(FromNow( $\left.30^{*} \mathrm{~min}\right)$ ); (End Session in 30 minutes

repeat \{Start of basic event checking loop\}

CheckEvents(Event, EventTime);

Case Event of

LeftBarBoxl: $\quad$ Reward( FeederBox 1);

RightBarBoxl: Plan( ToReward, FeederBoxl, FromNow $(3 * \sec )$ ); LeftBarBox2: Reward( FeederBox2);

RightBarBox2: Plan( ToReward, FeederBox2, FromNow( $3 * \mathrm{sec})$ ); end;

CheckChores;

until EndofSession; \{Boolean condition controlled by EndSession Chore\} end.

Figure 2. Example demonstrating the use of chores and the chore scheduler in an operant conditioning experiment. The program monitors and controls two two-bar Skinner boxes simultaneously. Text included in curly brackets is annotation for the benefit of the reader. 
Table 1

\begin{tabular}{cc}
\hline Case Event of & Reward( FeederBox1); \\
LeftBarBox1: & Plan( ToReward, FeederBox1, (FromNow $(3 * \sec )) ;$ \\
RightBarBox1: & Reward( FeederBox1); \\
LeftBarBox2: & Rew 1 (FromNow $(3 * \sec )$ ); \\
RightBarBox2: & Plan( ToReward, FeederBox2, \\
end; & \\
\hline
\end{tabular}

implemented by the "case" statement in the Repeat... Until loop at the end of the listing (Table 1).

To explain how these routines function, we explicate the main part of the program, line by line. The command InitESI initializes the ESI data structures and initiates the Timer's interrupt routines for polling the interface and checking the chore list. StartEventChecks initializes the hardware card, configuring 8 of its lines as input and 8 lines as outputs, and specifying the polling rate, or period, as $100 \mathrm{msec}$.

Plan and EndSession are the last two ESI scheduling procedures. Plan is used to schedule user-written chores (in this case one that causes the computer to signal the impending end of the experiment). EndSession determines the time at which the experiment actually terminates. When executed, EndSession performs two important functions. First, it stops the timer and terminates the interrupt routines in an orderly fashion (thus ensuring that the computer operates normally when the user program terminates). Second, it sets the ESI variable EndOfSession to TRUE, thus providing a convenient way of exiting the Repeat...Until loop, which follows.

The Repeat... Until loop is typically the core of any ESI User Program. Until EndOfSession becomes true, the program keeps cycling through this loop. The first command in the loop is CheckEvents, which updates the ESI variables Event and EventTime. If there are any events in the event buffer, the line number of the earliest one will be assigned to the Event, its time of occurrence will be assigned to EventTime, and the record of that event will be removed from the buffer. If the event buffer is empty when CheckEvents is called, Event will be set equal to zero.

The value given to Event determines whether any action is to be dispatched by the Case statement in Table 1 . For example, suppose that the rat has pressed the left bar in Box 2, which is connected to line 3 of the interface card. CheckEvents sets Event equal to LeftBarBox2 (previously established as a synonym for " 3 "). As a result, the Case statement causes the appropriate feeder to be activated.

Finally, the CheckChores command causes execution of all chores other than TurnOn and TurnOff, as explained below. If EndSession is triggered, the program terminates; otherwise, it cycles back to CheckEvents at the top of the loop.

\section{SCHEDULING CHORES}

\section{Syntax}

ESI's ability to schedule delayed chores is perhaps its most powerful feature and can be used even in the absence of an interface card. Every item in the chorelist has three components: (1) a number identifying the chore to be executed, (2) the time of execution, and (3) an integer argument (typically, but not necessarily, a line number to operate). These components are explicit in the scheduling procedure Plan:

$$
\text { Plan(ChoreID, Argument, Time) \{pseudo-code\} }
$$

The ChoreID is a unique constant assigned by the user. The ChoreIDs ToTurnOn, ToTurnOff, and ToEndSession are predefined in ESI, allowing the use of Plan to turn lines on or off or to end a session. The pairs of commands in Table 2 are equivalent.

Other ChoreIDs can be assigned by the user as described below. (NoArg is a predefined constant that can be used when no argument is to be specified, as in the case of ToSignalEnd in the example program.)

Time can be specified in several ways: in milliseconds from the current time (used in the example program); in milliseconds from the execution of InitESI; at a certain date and military time; and immediately. These modes are specified using the following function calls for the Time parameter:

FromNow (Milliseconds)

FromStart (Milliseconds)

OClock (Month, Day, Year, Hour, Minute, Second, Msec) Now

\section{The Scheduling Process}

When a scheduling procedure is called, ESI inserts the request into the Chore List. This list is checked once every millisecond to see if any chores have come due. If any intrinsic chores (TurnOn, TurnOff, and their "Planned" equivalents) are due, the timer executes them immediately. However, since the timer can only reliably execute tasks that are guaranteed to be of short duration, user-written chores (and EndSession) are executed only upon permission of the user program. This is the function of CheckChores and the procedure UserChores, which is therefore

Table 2

Equivalent Pairs of Commands

\begin{tabular}{lllll}
\hline Plan( ToTurnOn, & Line, & Time) & TurnOn( Line, Time) \\
Plan( ToTurnOff, & Line, & Time) & TurnOff( Line, & Time) \\
Plan( ToEndSession, & NoArg, & Time) & EndSession( & Time) \\
\hline
\end{tabular}


a necessary part of any ESI user program. If there is a UserChore that needs to be executed, ESI waits until CheckChores is called; then it updates the global variables ChoreID and Argument, and calls UserChores, which takes action based upon the value of the ChoreID.

Thus, if user-written chores are to be executed promptly and reliably, CheckChores must be called frequently enough, and each user-written chore must be assigned a unique ChoreID from 1 to 250. (In the example, this assignment was made just before the UserChores procedure.) Within this constraint, Plan can be used to accomplish almost any programmable task. UserChores can even schedule other chores, as in the case of ToSignalEnd, which, once triggered, reschedules itself repeatedly every 2 sec.

\section{GENERAL COMMENTS}

It is important to understand what ESI does not do, as well as what it does. ESI does not automatically store data in a permanent fashion (i.e., on disk). Because such input and output is usually the most time-consuming element in real-time programming, there is often a tradeoff between the amount of data processing and disk access to be done during the experiment versus the speed with which the user's program will be able to keep up with experimental events. We leave it to the user to decide how to strike the appropriate balance and to store data on disk in the desired level of detail. The event buffer does, however, provide the essential information needed to reconstruct experimental events: the number of the line that has gone on or off (the number is negative in the second case), and the time at which the event occurred.

Our convention for time stamping is also worth noting: ESI contains a function called MsecThisYear, which is responsible for the EventTimes given by CheckEvents. MsecThisYear is equal to 0.0 at the start of the year, and at the end of the year it is equal to $31,536,000,000.0$.

$$
\begin{aligned}
\frac{1,000 \mathrm{msec}}{\mathrm{sec}} & \times \frac{60 \mathrm{sec}}{\min } \times \frac{60 \mathrm{~min}}{\mathrm{~h}} \times \frac{24 \mathrm{~h}}{\text { day }} \times \frac{365 \mathrm{day}}{\text { year }} \\
& =\frac{31,536,000,000 \mathrm{msec}}{\text { year }}
\end{aligned}
$$

Since Turbo Pascal's real numbers are accurate to 11 significant digits, this scheme allows millisecond accuracy and provides a simple way to compute, and do real arithmetic on, for example, interresponse times, as well as to keep track of calendrical information. (A function is provided to convert MsecThisYear into conventional date and time.) It should be noted that the convenience of this technique comes at a price: real arithmetic is timeconsuming. However, if speed is a limiting resource, the use of a coprocessor can be considered.

ESI does not provide a full array of procedures for operant conditioning experiments (or any other specialized application). However, the beauty of Pascal is that such procedures are not difficult to write and, once written, can be reused in a variety of experiments. For example, Figure 3 provides a routine for the standard fixed inter- val (FI) operant conditioning contingency, in which a fixed amount of time must elapse before a response is rewarded. By including this code in a program above the UserChores Procedure, the user would only need to add one line to UserChores in order to be able to convert the experiment in Skinner Box 1 into a study of, say, choice between FI-15-sec and FI-25-sec schedules of reinforcement on the left and right bars, respectively.

Thus, the following line should be added to the Case statement in UserChores:

\section{ToSetUpNextReward:SetUpNextReward;}

Then, the Case statement in the main program could be modified as follows:

$$
\begin{aligned}
& \text { LeftBarBox1: FI }(15 * \mathrm{sec}) \text {; } \\
& \text { RightBarBox1: FI }(25 * \mathrm{sec}) \text {; }
\end{aligned}
$$

\section{PROGRAM DEVELOPMENT}

ESI contains three sets files for inclusion at different stages of program development. The alternative modes of operation they implement can greatly speed the development of more elaborate programs. The programmer selects the appropriate mode of operation by changing the compiler $(* \$ 1 .$.$) include directive at the beginning of$ the file before compiling.

The first set of include files comprises dummy procedures with headings and variable declarations identical to those of the working procedures, but with no statements. These files make compilation and syntactical debugging much faster. Once the User Program has compiled successfully, the $(* \$ 1 \ldots *)$ directive is changed to include the second set of routines, and the program is recompiled.

In debugging a compiled program, the second set of routines is useful. These provide an alternative hardware device driver that causes ESI to use the computer's keyboard and screen, rather than the interface board, as the devices for input and output. In this "simulated environment" mode, typing " $15+$ " on the numeric keypad can cause the program to act as if line 15 has gone on, and will write a message to that effect on the screen. If " $15-$ " is entered, the program will "think" line 15 has gone off. Similarly, if line 15 has been configured as an output line that ESI has been instructed to TurnOn, then a simulated output will be routed to the screen (rather than to the hardware interface card). Thus programs can be tested and debugged on any personal computer even if that machine has no interface card.

When a program is ready for running on a machine actually equipped with an interface card, the simulation routines are replaced by working routines and the program is compiled again. Within ESI, one more operating mode can be invoked using the command KeybdManOverride. In this "manual control" mode, typing " $15+$ " turns on the device connected to line 15 , and "15-" turns it off again. This can be used to control laboratory equipment manually and to override the User Program. 
(Include file for Fixed Interval Schedule of Reinforcement\}

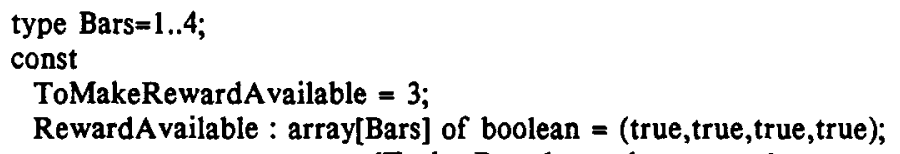

\{Turbo Pascal typed constant \}

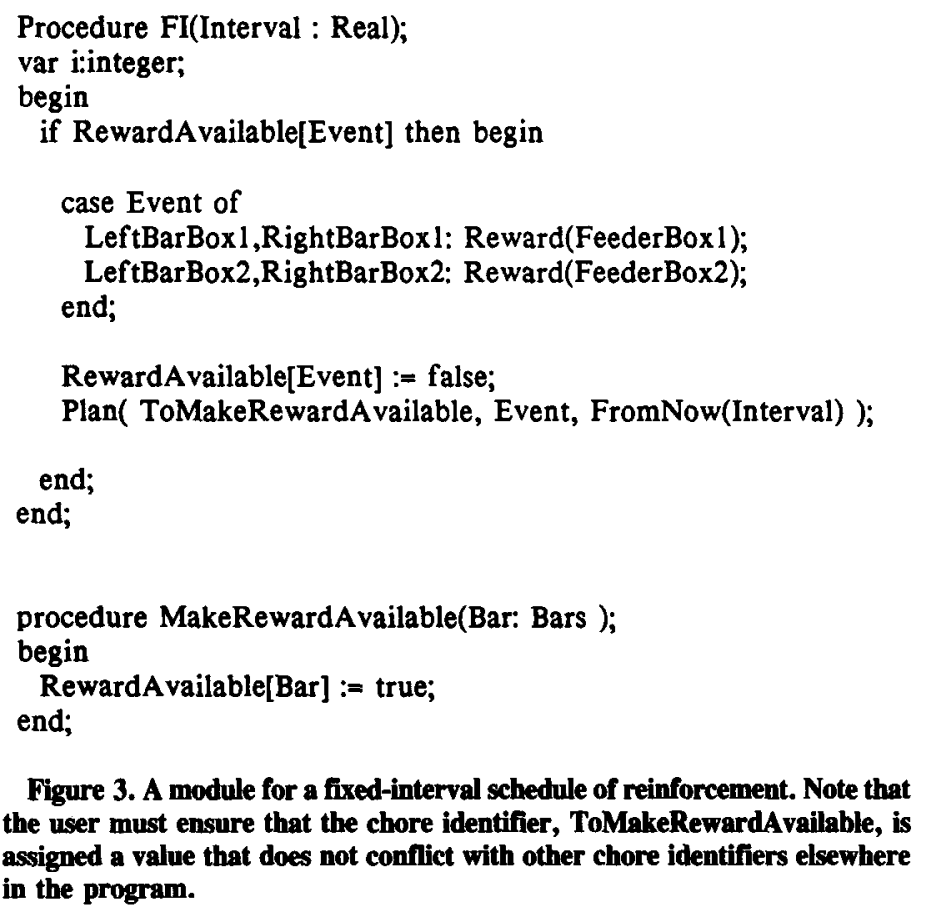

\section{DISCUSSION}

Our laboratory has been using a more limited Apple Pascal version of the system described above for several years. It has been successfully used for operant conditioning experiments of greater than average complexity (Schull et al., 1985) and in studies of circadian rhythms (Schull, Horvitz, Fiedler, \& McEachron, 1986). An implementation like the one described here has been working in our laboratory for several months. We hope that it will be of general utility and that it will be extended. Some possible lines of extension are outlined below.

The most pressing need is for device drivers to be written for other hardware interface cards. As noted, we have written a device driver for the DAISI interface card. This card can be used to control up to 72 input or output lines and can be purchased with optional rack-mountable optically insulated relay drivers and input modules for controlling or monitoring $28-$ and $128-\mathrm{V}$ devices. The hardware is not overly expensive, and we have found it to be highly reliable. However, other boards with similar capabilities are on the market at lower prices, and device drivers can easily be written to allow their use with ESI. We hope to act as a clearinghouse for such device drivers. Specifications for ESI device drivers will be made available, and we will provide assistance to those who are interested in writing device drivers for other cards.
Second, monitoring and control of both analog and digital inputs and outputs is within the capability of the DAISI card and of many other cards on the market. A valuable extension of the ESI system would be a set of analog tools. We are currently exploring this possibility and, again, encourage others to do the same.

Third, as the system comes to be used in a variety of applications, the need for additional tools (e.g., for highspeed data storage) may become clear. The ESI tools and the Pascal language are designed to make such expansions practical. If other users find the system as useful as we have, such extensions are inevitable and welcome.

\section{REFERENCES}

KaPlan, H. L. (1985). Design decisions in a Pascal-based operant conditioning system. Behavior Research Methods, Instruments, \& Computers, 17, 307-318.

Schull, J., Gelch, H., Vitale, J., Allen, A., James, S., \& HarRson, M. (1985, August). Optimal foraging in depleting patches: Operant simulations compared with seminatural observations. Paper presented at the Animal Behavior Society Meetings, Raleigh, NC.

Schull, J., Horvitz, J., Fiedler, L., McEachron, D. L. (1986, November). Effects of thyroparathyroidectomy and lithium on circadian activity rhythms in rats. Paper presented at the meetings of the Society for Neuroscience, Washington, DC. 